\title{
Computer-Integrated Manufacturing System for Membrane Structures
}

\author{
Kazuo Yokobori ${ }^{1, *}$, Tomo Miura ${ }^{2}$ \\ ${ }^{1}$ Taiyo Kogyo Corporation, Engineering Division, 4-8-4,Kigawa higashi, Yodogawa-ku,Osaka 532-0012, Japan \\ ${ }^{2}$ Taiyo Kogyo Corporation, Information Department, 4-8-4,Kigawa higashi, Yodogawa-ku,Osaka 532-0012, Japan
}

\begin{abstract}
A membrane structure is a space structure composed of a membrane material (fabric or film), cables, and steel frames, among others. It reduces the environmental load for transporting materials and constructions; for instance, compared with conventional roofs that have steel panels or tiles, the membrane structure of a roof is lightweight. Computer analysis and three-dimensional (3D) models are required for determining the stable shape of such tensile structures. It is useful to use computer-integrated systems for the design and manufacturing process because these 3D models consist of numerical data. In this study, we developed a system program based on artificial intelligence methods, with a support vector machine instead of human judgment for the membrane structure estimation and for a probabilistic optimization to predict the differences caused by production loss etc. and compare the results after actual production. And we got close predicted results to the person.
\end{abstract}

\section{Introduction}

Compared to other structural forms, membrane structures are lightweight and have a reduced environmental impact of manufacturing, transportation, construction, maintenance, and disposal. Membrane materials (resin-coated fabrics and films) with more than 20 years of useful life have been developed for permanent buildings and the expansion of their use can contribute to the creation of continuously updated urban spaces.

It is necessary to improve their functionality and productivity and to develop a design both beautiful and cost-effective for the customers to make these structures easy to use.

In recent years, the Enterprise Resource Planning (ERP) system has become a popular tool for the improvement of productivity. The ERP system facilitates a company to make an action plan by managing the following items on the computer.

(1) Amount of raw materials required (Material procurement).

(2) When and where to buy, store, and manufacture raw materials, to keep costs low and to meet deadlines (Process Management).

(3) Production costs and selling price associated with (1) and (2) (Cost estimation).
It is important to determine (3) early so that, if a reasonable cost is forecasted, an accurate quotation can be presented to the customer for negotiation.

To operate, the ERP system requires detailed dimensions and parts of the configuration data of the product in the construction field, which are imported from a building information modeling data source. Such information is conveyed better by three-dimensional data. However, in the Japanese building industry, there are still a lot of two-dimensional (2D) CAD drawings, while only threedimensional (3D) ones are just a few.

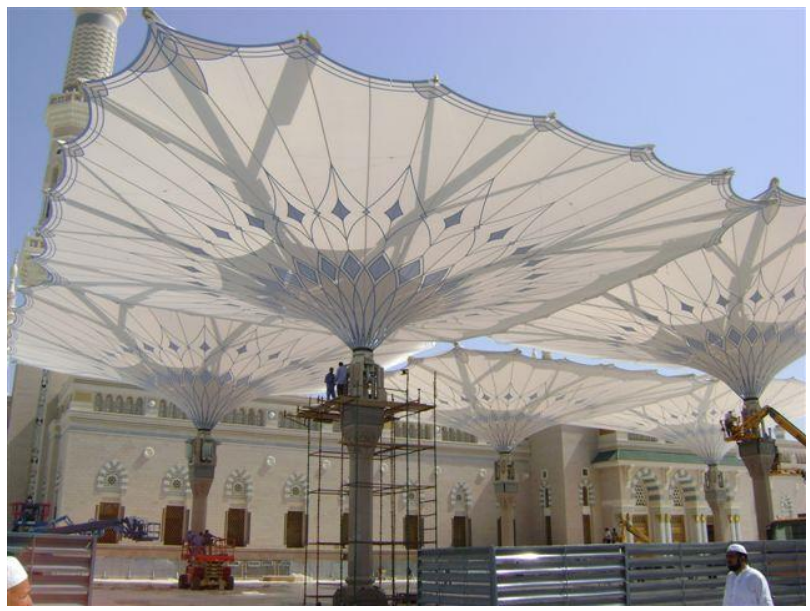

Fig. 1. Madinah Haram Piazza Shading.

\footnotetext{
* Corresponding author: yk002500@mb.taiyokogyo.co.jp
} 
General Contractor : Saudi Binladin Group Concept and Architectural Design \& Engineering : SL-Rasch Manufacturing and on-site Installation of the large-scale umbrella system : Liebherr Intertrading Membrane fabrication: Taiyo Kogyo Corporation

Therefore, there is a lack of accuracy in the ERP input data, which makes the manufacturing management difficult.

On the other hand, Taiyo Kogyo Corporation is a manufacturer of membrane structures (Figure 1) that requires $3 \mathrm{D}$ analysis of many properties to determine the peculiar surface shape of its products.

We developed a computer program to calculate the important information from the $3 \mathrm{D}$ analysis of data automatically, used them to operate the ERP system, and verified the difference between generated and actual production data.

The purpose of this study is to accurately predict the actual production data of the membrane structure, and to promote the use of the membrane structure which is a structure form with a little environmental load.

\section{Membrane structure design}

To understand the membrane structure, we will explain the $3 \mathrm{D}$ analysis that allows the easy determination of its peculiar surface shape.

Many buildings are constructed based on the sizes and shapes entered in the CAD project; however, tension stable structures such as the membrane structures require a numerical analysis, called shape-finding analysis, to determine the resulting shape in advance. Given the use of commercial software for such a purpose, we developed it long ago and used it in the in-house programs $[1-4,6]$.

First, as Figure 2 shows, we create an analysis model consisting of membrane, beam, and cable elements in a straight line.

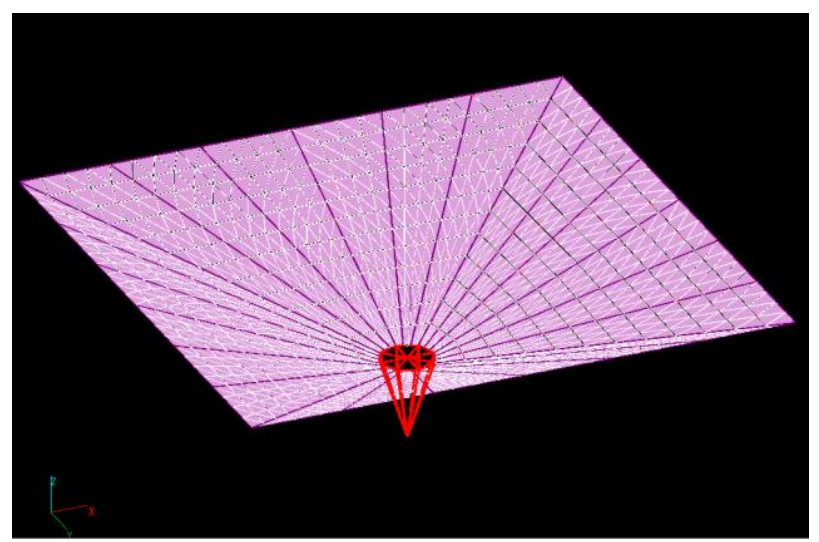

Fig. 2. Modeling shape.

At this stage, the shape is made up of straight lines and a plane. Since they are easily deformed by external forces, the membrane and cable materials do not have stable shapes when no tension is applied. Hence, it is necessary to introduce some tension during the construction.

The final shape is determined by analyzing the deformation associated to the tension of cables and membranes, which is kept constant. This is the shapefinding process.

Although the stress of the structural materials varies depending on the deformation in the general structural analysis, that of the membrane and the cable materials remains constant in the shape-finding and the analysis determines which load and stress deform the shape to the position to match. As Figure 3 shows, after the shapefinding, the modeling shape becomes a specific 3D surface of the membrane structure.

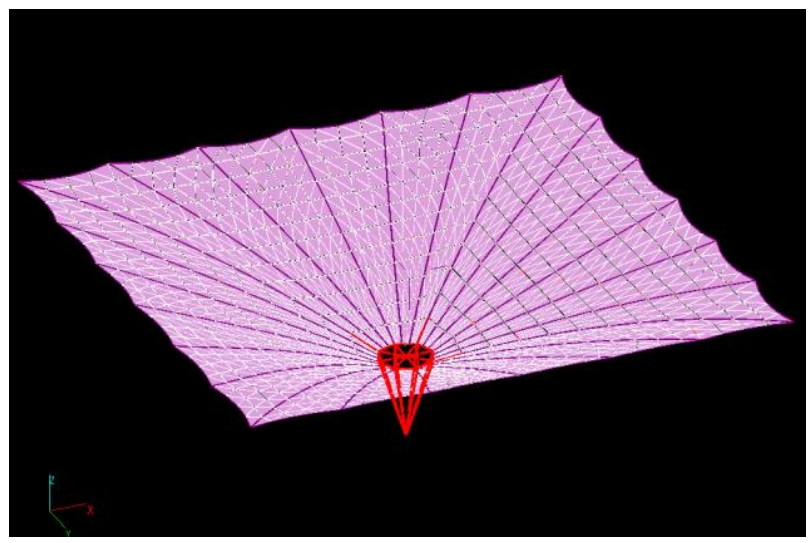

Fig. 3. Modeling shape after shape-finding.

By adjusting the tension ratio between cable and membrane materials (warp and fill direction, respectively), the curved shape is controlled and the tension ratio and values are approximated to those of the design image.

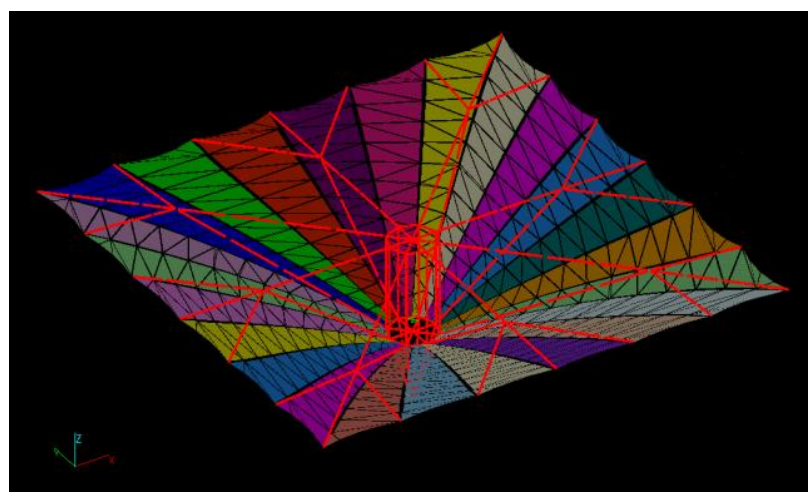

Fig. 4. Modeling shape split into planar parts (top view).

Once the shape is determined, the curved surface is subdivided into a planar deployable mesh (Figure 4) to cut out the plane parts from the membrane material. This process is called patterning [5]. The shape so obtained is used to calculate the amount of the steel material and to manage the cost forecasting and the manufacturing process.

The entire procedure is done manually using the inhouse developed system and it is time-consuming.

\section{Design Automation}

The purpose of this study in the future is to make the design work more efficient by applying it to the actual design. 
In the actual design, if the characteristics of the product do not meet the requirements established before the production, the damage and cost associated to the successive correction are huge: therefore, we must be cautious with the automation of the process.

However, since most of the buildings are only one custom-made product, what is the manufacturing cost of this structure? At present, the estimation of the cost is done repeatedly manually with a simple trial of the design and manufacturing process.

In this study, we are trying to automate this estimation process.

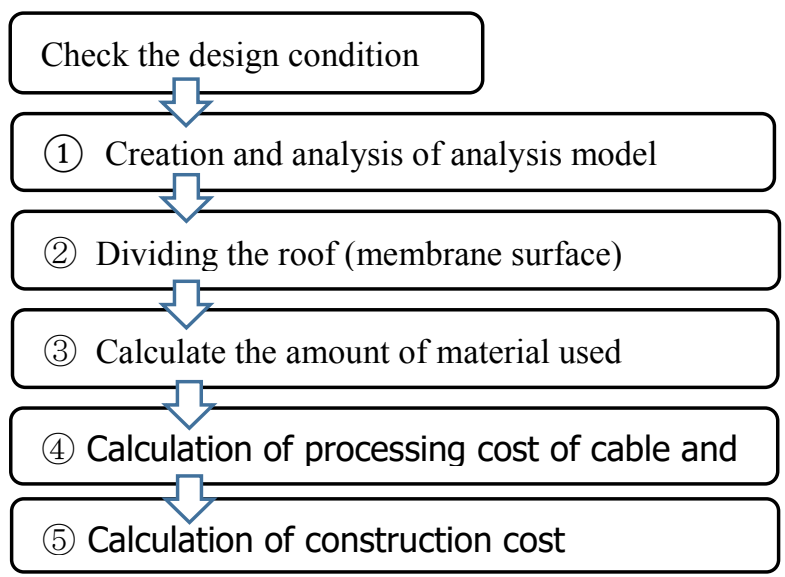

Fig. 5. Cost estimation steps.

It is difficult to check the design conditions automatically and to perform the model generation and the analysis (Figure 5, (1)); this step will represent a challenge for the future developments.

However, the steps (2) and (3), after the design, has been decided according to the following points.

(1) Dividing the roof in (2) and reducing the amount of use in (3).

(2) Placing the parts on the cloth in (3).

Clarifying the mechanism to determine the rules for the (1) and (2) tasks will enable the automation of (2) and (3) and the consequent streamlining of the manufacturing.

The calculations in (4) and (5) can be performed easily with the standard specifications and the unit price in conjunction with the ERP system.

\subsection{A program to do the human work}

Figure 6 shows an example of a bird's eye view and the constituent parts of the membrane roof model. It is useful to determine the amount of cloth required for such a model, to examine the human work procedure, and to create a prediction program to reproduce the human process and compare the results (to be used in the ERP). $[7,8]$

In the production, the different elongation of the fabric material in the warp (roll winding direction) and fill directions must be considered.

In addition, the roll width of the fabric is about 1-4 m and when the parts split, the warp direction is matched and the membrane is divided and arranged to fit in this width.

Figure 7 shows the result of this automatic arrangement by prediction program. The dotted line portions in Figures 5 and 6 refer to the same region. Such region is divided into three because it would not fit two divisions in the fabric width.

As Figure 8 shows, the actual amount of fabric used in the model was determined and compared with the values estimated manually (reference) and by the prediction program.

Each of the A-H values in the Figure 8 indicates a property based on the actual value and its percent difference from that.

Because the fabric width is different depending on the material type, the horizontal axis is used fabric length $\times$ fabric roll width $=$ used fabric amount $\mathrm{m}^{2}$. However, there is no manual (human) estimation for the values $G$ and $\mathrm{H}$.

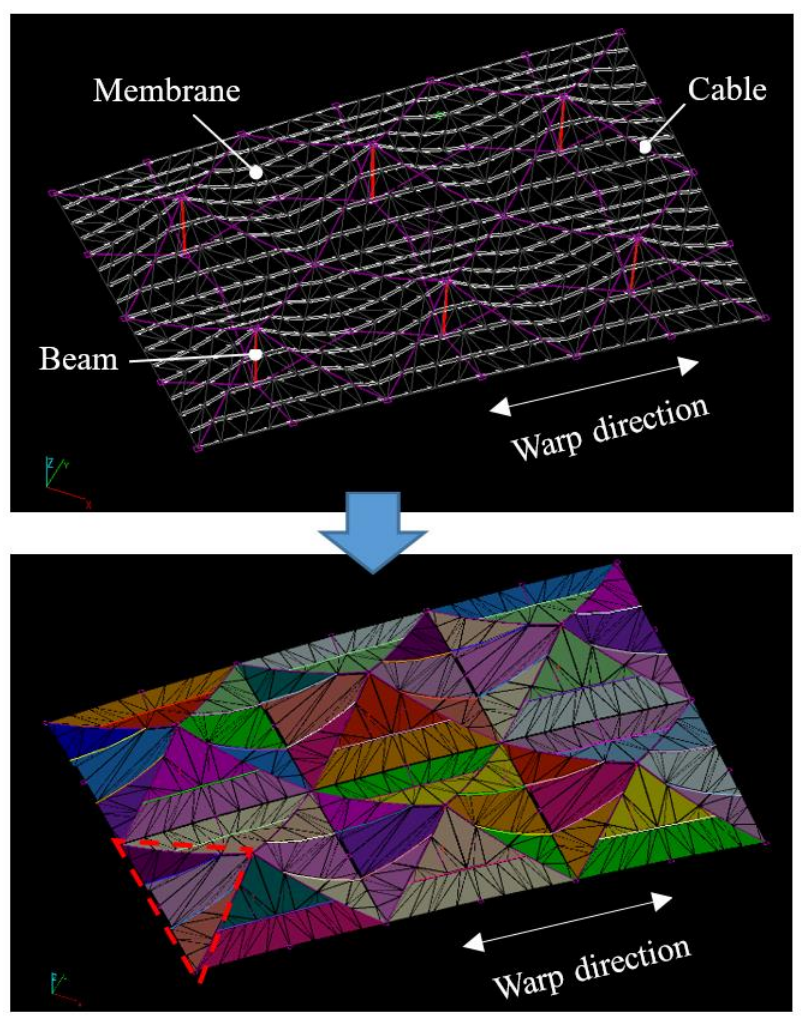

Fig. 6. Automatic separation of the membrane roof according to direction and width of the fabric.

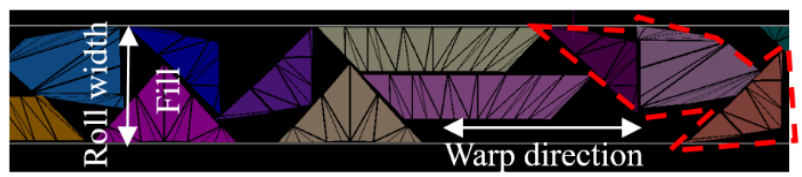

Fig. 7. Roof divisions placed on the fabric roll. 


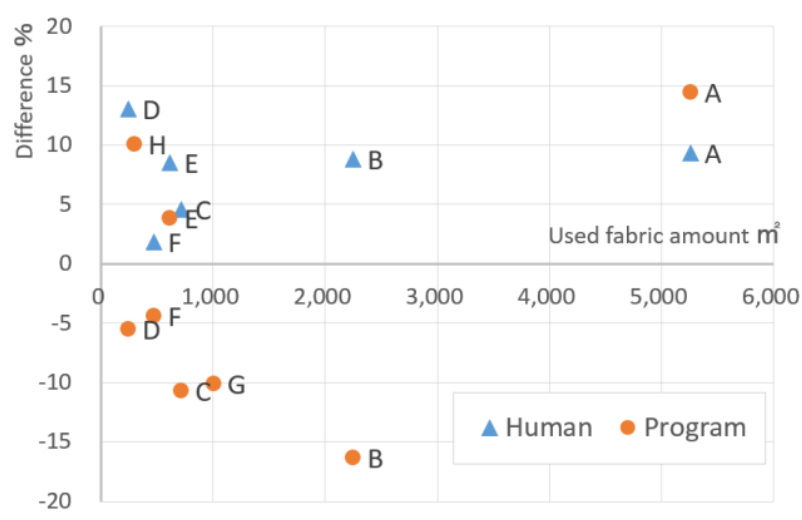

Fig. 8. Comparison between the actual, human estimated, and software predicted amounts of fabric used in the model.

In Figure 8, the values predicted by the program are larger than the human estimated ones, while the difference from the actual values is lower.

\subsection{Improvement of the prediction program}

The cause of the differences shown in Figure 8 is considered below.

\subsubsection{Branch of human processing}

Figure 9 presents a case to examine the cause of the different results. It is the same example shown previously in Figures 6 and 7 to examine the processing data of the factory (used to control the automatic cutting machine), and the dotted line portion indicates the same region of the other two figures.

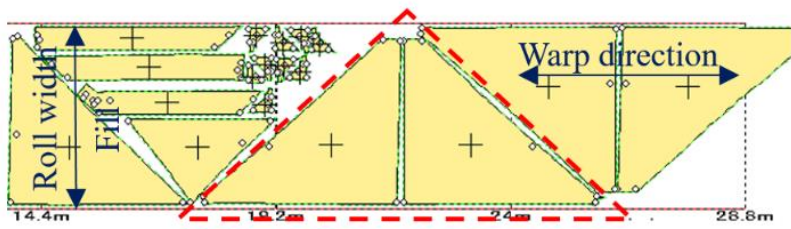

Fig. 9. Factory processing data.

Although such region was automatically divided into three parts in Figure 6 and 7, in Figure 9, it is divided into two parts manually. By looking closely, the corner part of the triangular area (upper left of the dotted line portion in Figure 9) appears further divided. This division reduces the void of the fabric and improves the yield, but also causes the differences in Figure 8.

Figure 10 shows the typical membrane structures for a better understanding.

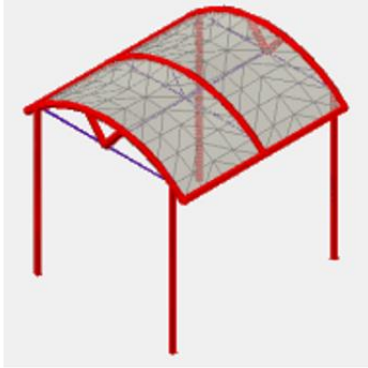

Frame type

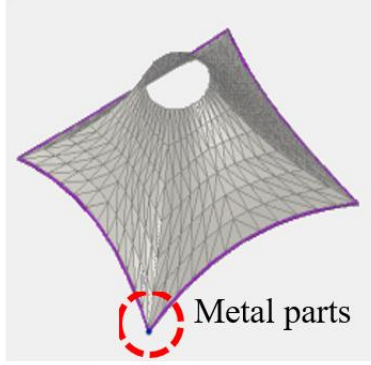

Suspension type
Fig. 10. Typical membrane structures.
In the frame type, the further division of a corner portion and its effect are not clearly visible. Regarding the suspension type, because the drawing metal parts of the corner are attached to the membrane structure (the dotted line portion in Figure 10), there is no sense of discomfort even when designing different parts.

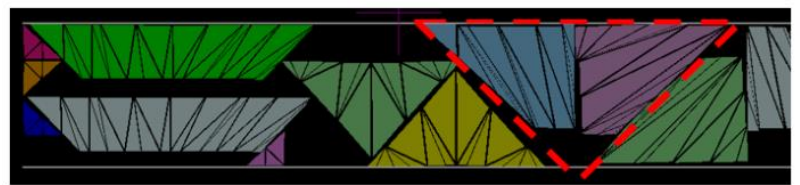

Fig. 11. Automatic placement on the fabric roll of the suspension type after the automatic separation of the corner part of the dotted line portion.

Figure 11 shows an example of the execution of the prediction program based on the specific separation of the suspension type.

Compared to Figure 11 (before the improvement), the unused portion is reduced and the fabric is used more effectively.

Different factors should be considered in the estimation of the frame and the suspension type. Even in cases where one factor cannot be clearly determined, human beings still have to establish both; hence, we tried to introduce a support vector machine for this decision.

In addition, the design in Figure 12 shows the aligned position of the dividing line and good evenly splits, resulting from some good human decisions.

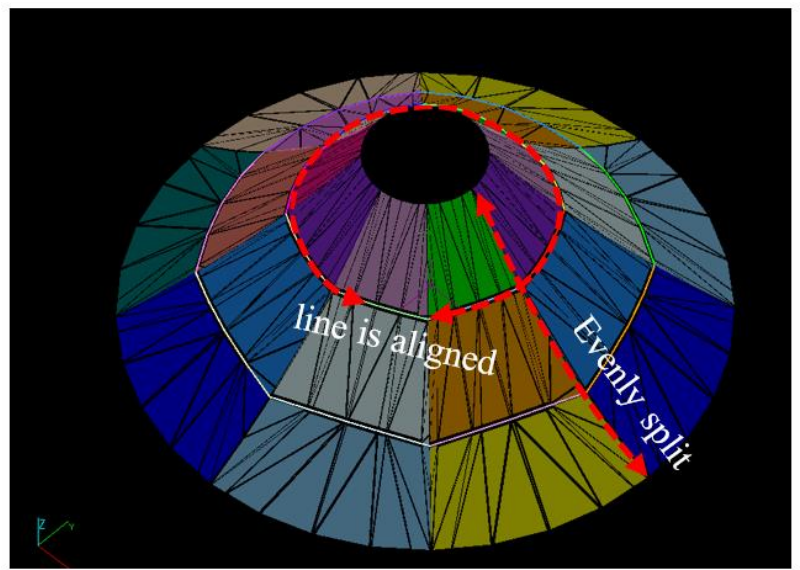

Fig. 12. Favorable design decisions.

Such human decisions cause the difference with the results obtained from the prediction program. These judgments are difficult to standardize and quantify, and specific values and threshold are hardly identified. In a traditional program that switches processing in a conditional branch, it seems difficult to obtain the result by such decisions, which sound reasonable even in a vague case.

In this study, we used the method of the support vector machine based on the ratio of the demand for the design property, the membrane-cable-steel materials; then, we formulated an individual judgment and calculated the fabric length to use for it. [9] 


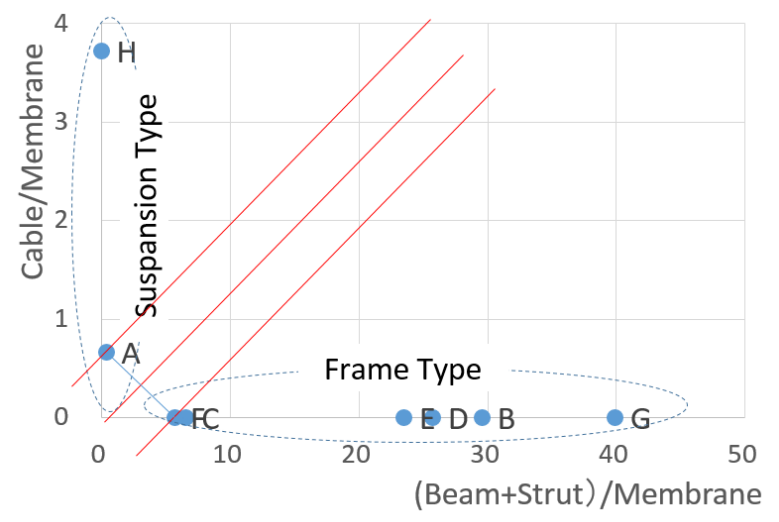

Fig.13. Results of human decision combined with a support vector machine.

For example, if you want to change the processing of the suspension and frame type structures, it is very difficult to have the program making such decision based on the difference in dimensions and shape, without risking that it stops on an error in a vague case. In such a situation, the index to combine a characteristic amount in the judgment by the artificial intelligence (AI) method, called support vector machine, is used to determine the type from the region on the graph in Figure 13.

The processing is lighter than the neural network and the deep learning (of vogue now), and it is possible to make a decision even in an ambiguous case.

In this example, the cable/membrane or (column beam + strut)/membrane weight ratio is plotted on the graph and its margin divides the closest contact between each other to determine the threshold value of this decision.

\subsubsection{Prediction of undecided items in the early stages}

The actual value fluctuates due to the presence of undecided items in the estimation stage (of the construction costs, by customer request). As Figure 14 shows, in the actual case (in which the amount actually used in the factory was entered in the ERP system), except for (A) the amount of the divided membrane surface parts, all the other amounts are unknown at the estimation stage: B) increase or decrease by design change, (C) accessory parts of the details, (D) cutting impaired, etc.

(B) refers to the design change during the actual building of the project, which might lead to a change in the dimensions.

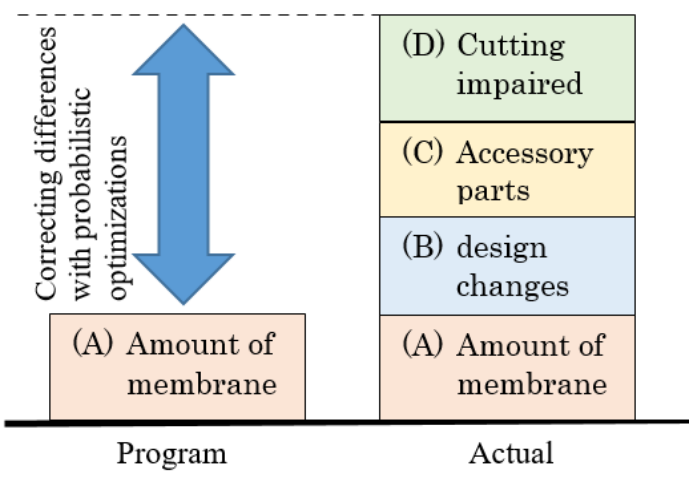

Fig.14. Unknown amounts at the estimation stage.
(C) indicates the size of the details for the membrane surface; the frame joints are determined in the detailed design step and represent the accessory parts. This amount could be counted; however, it cannot be done with our prediction program due to other unknown amounts in the estimation stage.

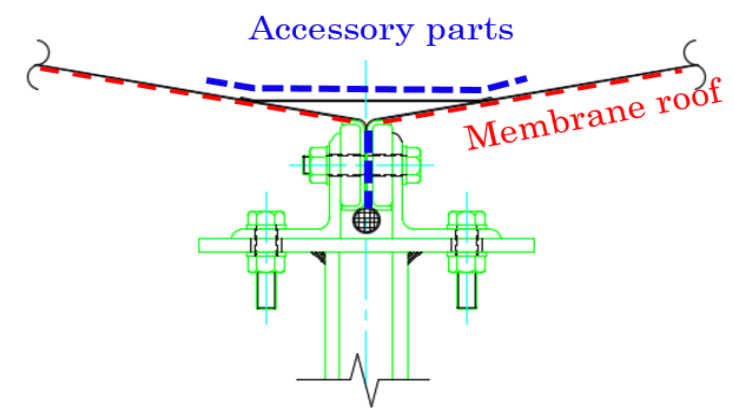

Fig. 15. Accessory parts in detail.

(D) refers to the cutting impaired and etc. in the stage of cutting parts from the fabric roll; it is caused by practical issues related to a usage larger than planned of the automatic cutting machine.

The unknown amounts of (B)-(D) are difficult to consider in the estimation stage; however, they are required for the estimation results.

In this study, we used probabilistic optimization methods to predict these values. [10]

The amount of fabric used in Section 3.2.1 is $\mathrm{x}_{\mathrm{i}}$, the actually used length is $y_{i}$, the loss function is given from past results (actually used length) and by Equation (1), which is determined to be a minimum of $\mathrm{A}$ and $\mathrm{B}$. $f\left(x_{i}\right)$ is chosen as the predict value $y_{i}$

$$
\sum_{i=1}^{n} l\left(y_{i}, f\left(x_{i}\right)\right) \quad, \quad f\left(x_{i}\right)=\left(1+\left(A \log x_{i}+B\right)\right) x_{i}
$$

" $A \log \left(x_{i}+B\right)$ " in Equation (1) to show the difference with the actual values of fabric usage $x$ of the horizontal axis in Figure 8.

At this time, we calculated A and B from a certain number of existing samples; however, we are developing a sequential learning method to update A and B for each new property data obtained on the ERP system in the future.

The results of the predicted value $y_{\mathrm{i}}$ of the amount of fabric used were processed and compared with the manual estimated values in Figure 16.

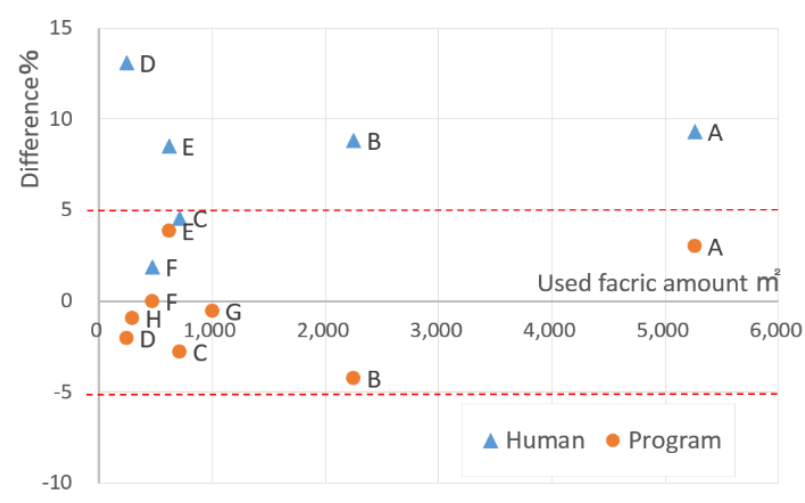

Fig. 16. Comparison of actual, human, and program predicted (after improvement) values of fabric used. 
The predicted amount of fabric used was improved to fit within $\pm 5 \%$ of the results, which is closest to the actual values than the human estimated amounts.

There are some problems induced in the estimation manual processing, as listed below.

- Differences in parts not standardized for each person.

- Possibility that human error occurs repeatedly.

The automation of this procedure is of great importance, because it is a quite time-consuming process when performed manually

Some of the examples presented in this work were actually built and the cost information (based on actual design and production results) were recorded in the ERP system. These records allowed us to improve the system and, if a good method of accuracy will be found, it could be immediately used for the improvement in the accuracy of the actual estimation work and for the automation of the design and manufacturing process in the future.

\section{Conclusion}

In this study, we outlined the difficulties associated to a program that mimics the processing of a human operator (according to a clear judgment standard), in particular:

(1) The criteria adopted by the human operator are not clear and are hard to standardize;

(2) Determining unforeseen circumstances.

We used the support vector machine to determine the effect of unknown quantities on the predicted results in the estimation phase.

The accuracy of the prediction program could be further improved by probabilistic optimization methods combined with past actual values recorded on the ERP system.

To proceed with the improvement of this automation work, our next steps will be based on answering the following questions:

- How guesses by probabilistic optimization methods will change according to the data recorded on the ERP system?

- Is it practical to use AI technology to handle judgments that are based on unclear operator's criteria?

\section{References}

1. T. Miyamura, H. Noguchi, K. Yokobori, Proceedings of the Japan Society of Mechanical Engineers, Bifurcation analysis of wrinkles in membrane structure by Element Free Galerkin Method, 10th Computational Mechanics Conference, p447-448,(1997)

2. T. Miyamura, H. Noguch, K. Yokobori, Proceedings of ICCS '97 in Taiwan, Geometrically Nonlinear
Analyses of Membrene Structures by Using Element Free Galerkin Method, , (1997)

3. H. Noguchi, K. Yokobori, Proceedings of Japan Society for Computational Engineering and Science, Analysis of membrane structure by geometrical nonlinear Element Free Galerkin method, No.2 p387-390, (1997)

4. T. Miyamura, H. Noguchi, K. Yokobori, Proceedings of the Japan Society of Mechanical Engineers, Formulation of geometrical nonlinear Element Free Galerkin method and its application to analysis of membrane structure, File A 64(632) p1753-1760, $7(1998)$

5. T. Kawashima, S. Yahashi, H. Noguchi, K. Yokobori, Proceedings of Membrane structure association Japan, Mesh-less geodesic search on arbitrary surfaces by moving least squares method, weblink, (1999)

6. T. Kawashima, H. Noguchi, K Yokobori, Proceedings of Membrane structure association Japan, Mesh-less analysis for design of cable reinforced membrane structure,weblink, (2000),

7. Kazuo Yokobori, Summaries of Architectural Institute of Japan, Automatic accumulation from membrane structure analysis model, Information Systems Technology, 11042, (2017)

8. Kazuo Yokobori, Tomo Miura, Proceedings of The Japan Society for Artificial Intelligence, AI utilization for membrane structure design and manufacturing, AI Applications-Industrial applications(3), 2M2-03, (2018)

9. Ichiro Takeuchi, Karasuyama Masayuki, Support vector Machine (machine learning Professional Series) Kodansha Ltd., (2015)

10. Taiji Suzuki, probabilistic optimization (machine learning Professional Series) Kodansha Ltd., (2015) 